\title{
Estudio dosimétrico de la embolización endovascular de varicocele en pacientes pediátricos
}

\author{
Calama Santiago JA, Penedo Cobos JM, Molina López MY, González Ruiz C, García Mollá R, \\ Sierra Díaz F.
}

Servicio de Dosimetría y Radioprotección. Hospital General Universitario Gregorio Marañón. Madrid.

Actas Urol Esp. 2008;32(8):833-842

\section{RESUMEN}

ESTUDIO DOSIMÉTRICO DE LA EMBOLIZACIÓN ENDOVASCULAR DE VARICOCELE EN PACIENTES PEDIÁTRICOS

Introducción y objetivos: El varicocele es una anomalía benigna que se detecta generalmente en pacientes jóvenes, y para cuyo tratamiento existen técnicas quirúrgicas. La embolización endovascular es un procedimiento terapéutico alternativo mínimamente invasivo, pero con un riesgo radiológico asociado que resulta esencial conocer para valorar el método de tratamiento más adecuado. El objetivo del trabajo es determinar niveles locales de dosis recibidas en embolizaciones de varicocele en pacientes pediátricos y minimizarlas, estimando el riesgo radiológico.

Material y métodos: El estudio se ha realizado en dos fases; una retrospectiva para 15 embolizaciones en pacientes con edades comprendidas entre 10 y 18 años, sin intervención previa en el método de trabajo, y otra prospectiva para 10 pacientes de 10 a 16 años de edad, utilizando algunas técnicas de reducción de dosis implementadas en el equipo. Se conocía el tiempo de escopia y el valor del producto dosis-área de cada intervención. Además, en la fase prospectiva, se han utilizado dosímetros termoluminescentes para la evaluación de la dosis máxima de entrada en piel, y películas radiográficas para verificar la zona irradiada.

Resultados: La dosis efectiva media estimada en el estudio retrospectivo fue de $8.8 \mathrm{mSv}$. El riesgo total medio de inducción de cáncer fatal en cualquier localización fue un $0.16 \%$, siendo un $0.0007 \%$ para los efectos hereditarios. La dosis máxima de entrada en piel fue de 250 mGy, siendo 2 Gy el umbral de dosis para la aparición de efectos deterministas en piel. Para los pacientes del estudio prospectivo, las dosis y el riesgo radiológico se redujeron en más de un $70 \%$.

Conclusiones: La estimación de riesgo radiológico estocástico es relativamente elevada comparada con el riesgo de complicaciones severas en las intervenciones quirúrgicas de varicocele, excepto para el caso de efectos hereditarios. Son descartables los efectos de tipo determinista, salvo en complicaciones del procedimiento. La exposición a la radiación debe ser optimizada. Es necesario que los clínicos prescriptores e intervencionistas conozcan los riesgos radiológicos asociados a la intervención e informen a sus pacientes.

Palabras clave: Varicocele. Embolización. Dosis efectiva. Riesgo estocástico. Dosis gónadas. Riesgo hereditario.

\section{ABSTRACT}

PAEDIATRIC VARICOCELE EMBOLIZATION DOSIMETRIC STUDY

Introduction and objectives: Varicocele is a benign condition, often asymptomatic, that appears among young men with a normal life expectancy. The endovascular embolization is a minimally invasive therapeutic procedure indicated for varicocele treatment, although there are safe surgical alternatives. It is essential to know the radiological risk associated with the procedure. The aim of this work is to determine local dose levels in paediatric varicocele embolization, in order to minimize and to estimate the radiological risk.

Material and methods: In the retrospective study, 15 cases were analyzed, all of which were paediatric patients (1018 years). The screening time and the dose-area product were known, as provided by the X-ray equipment. In the prospective study, dose optimization techniques were implemented, and in addition, radiographic films were used in order to verify the radiated zone and termoluminiscense dosimeters in order to evaluate gonad dose and maximum skin dose for 10 paediatric patients (10-16 years).

Results: In the retrospective group, the average effective dose was $8.8 \mathrm{mSv}$. The total average risk of a fatal cancer induction in any location was $0.16 \%$, and $0.0007 \%$ for hereditary effects. The maximum skin dose was 250 mGy, which is far from the threshold for deterministic effects ( 2 Gy). In the prospective group, dose and radiological risk decreased by more than $70 \%$.

Conclusions: Hereditary effects were very low in contrast to the fatal cancer risk estimation. According to the results we can discard deterministic effects, unless complications appear in the procedure. Radiation exposure must be optimized. It is necessary that medical staff know the radiological risks associated with the intervention, and inform the patients about them.

Keywords: Varicocele. Embolization. Effective dose. Stochastic risk. Gonad dose. Hereditary risk. 
$\mathrm{E}$ varicocele es una dilatación varicosa de las venas del plexo pampiniforme que drenan los testículos. Este trastorno afecta aproximadamente a un 15\% de los varones jóvenes, siendo clínicamente relevante en un 5-20\% de los pacientes, en los cuales, viene acompañado de dolor o está relacionado con atrofia testicular e infertilidad ${ }^{1,2}$. En estos casos está indicada la intervención, que consiste en seccionar o detener el flujo de la vena espermática, ya sea mediante extirpación quirúrgica, extirpación laparoscópica o embolización percutánea.

En 1978 Lima et al. ${ }^{3}$ publican el primer artículo sobre la terapia percutánea como técnica para el tratamiento del varicocele. Esta intervención se efectúa realizando una incisión puntiforme en la vena femoral, a través de la cual, se guía un microcatéter hasta la zona afectada por medio de un equipo de fluoroscopia. Se realiza seguidamente una flebografía de la zona mediante inyección de contraste para delimitar las venas afectadas, y se ubican "coils" metálicos por medio del catéter para bloquear el flujo venoso de las venas patológicas, derivándose la sangre hacia venas normales.

La embolización tiene ciertos beneficios clínicos respecto a la cirugía convencional y a la laparoscópica, ya que se trata de un procedimiento mínimamente invasivo, sin anestesia general, que requiere un tiempo de intervención y hospitalización generalmente corto. Además, el paciente puede llevar una vida normal desde el día siguiente a la intervención, y realizar actividades físicas en menos de una semana. Como contrapartida aparece el riesgo adicional del uso de radiación ionizante. Teniendo en cuenta que la patología es benigna y lleva asociada una esperanza de vida normal, y que existen alternativas quirúrgicas, es esencial que la dosis recibida por el paciente durante la intervención sea conocida y minimizada.

El uso de radiaciones ionizantes como técnica médica lleva asociado un riesgo de aparición de efectos de tipo estocástico o probabilístico, especialmente relevante en el caso de pacientes pediátricos. Tanto la Comisión Internacional de Protección Radiológica (ICRP) ${ }^{4}$ como la National Radiological Protection Board (NRPB) ${ }^{5,6}$ han evaluado los riesgos de mortalidad por cáncer radioinducido por unidad de dosis efectiva en función de la edad a la que se produce la exposición, y muestran un aumento del riesgo al disminuir ésta. Este aumento se debe a una mayor radiosensibilidad de los tejidos de estos pacientes, así como a una mayor esperanza de vida, y por tanto, a un riesgo potencial de manifestación de efectos nocivos más elevado. Adicionalmente, en embolizaciones de varicocele, debido a la dosis que reciben los testículos, pueden aparecer también otro tipo de efectos estocásticos como son los efectos hereditarios en la descendencia.

Determinados procedimientos fluoroscópicos pueden llegar a superar los umbrales para la aparición de efectos deterministas, debido a las altas dosis utilizadas. La ICRP- $85^{7}$ describe la embolización percutánea de varicocele como una técnica fluoroscópica de dosis media (decenas de mGy de dosis absorbida en piel), excluyendo la aparición de efectos deterministas como la esterilidad, temporal o permanente, y las lesiones cutáneas.

Aunque se han publicado estimaciones de dosis y análisis de riesgos en adultos sometidos a embolizaciones de varicocele, la bibliografía sobre las dosis recibidas en este tipo de procedimiento en pacientes pediátricos es escasa. Nosotros realizamos un primer estudio dosimétrico de 15 intervenciones, con el fin de establecer niveles de referencia locales de dosis para poder aplicar, posteriormente, métodos de optimización y reducción de dosis en un estudio sobre 10 pacientes.

\section{MATERIAL Y MÉTODOS}

Se realizó un estudio dosimétrico de embolizaciones endovasculares en pacientes pediátricos desde enero de 2005 a diciembre de 2007 en la sala de Hemodinámica Infantil del Hospital General Universitario Gregorio Marañón de Madrid. Se recogieron datos relativos a la técnica de exploración: el potencial medio del tubo $(\mathrm{kV})$, el tiempo de escopia y de cine, el número de series y de imágenes realizadas, y el producto dosis-área (PDA). En el estudio prospectivo se midieron, además, las dosis de entrada en piel y en gónadas mediante dosímetros de fluoruro de litio (TLD-100). En todos los casos se disponía de parámetros que pueden relacionarse con la estimación de dosis recibida, como la edad, el peso o la altura.

La embolización se llevó a cabo con sedación, accediendo por vía percutánea transfemoral derecha y mediante la cateterización de la vena renal izquierda. Tras inyectar contraste radiológico para localizar la vena espermática, se procedía a su 
embolización colocando dos "coils", a la altura del canal inguinal y de la vértebra L3, hasta conseguir la oclusión venosa total, como se muestra en la Figura 1. Todos los procedimientos fueron realizados por el mismo clínico, sin influir en su método de intervención durante el desarrollo del estudio.

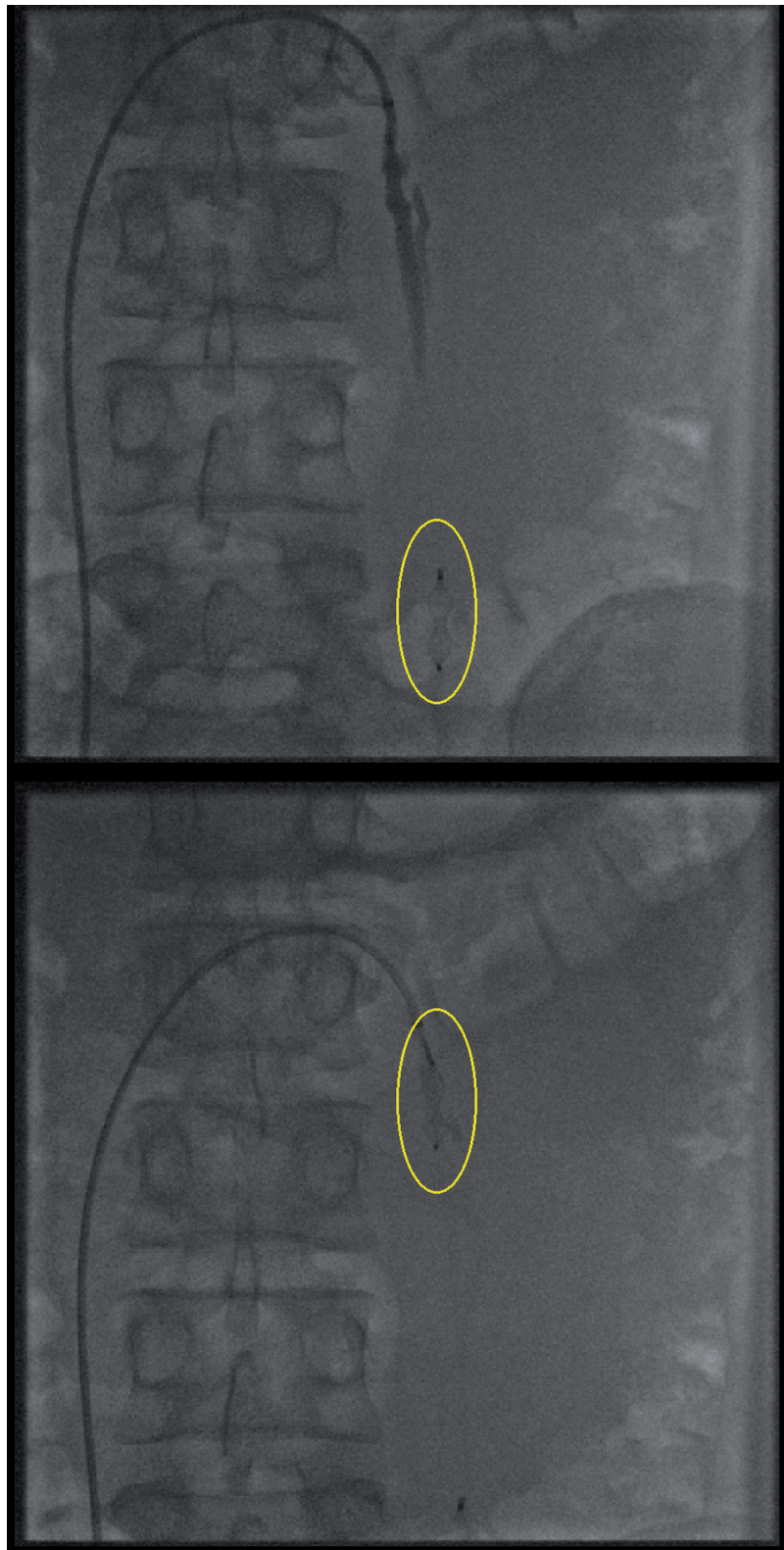

FIGURA 1. Ubicación de los elementos embolizantes en la vena espermática interna.

\section{Equipo digital}

Se utilizó un equipo digital de rayos $\mathrm{X}$, marca General Electric modelo INNOVA 2000, con un generador de alta frecuencia VAMP de $100 \mathrm{~kW}$ y un tubo de rayos X Maxiray 150 versión 5 de doble foco de
0.6 y $1.2 \mathrm{~mm}$. El detector digital tenía un tamaño de 20.5 × $20.5 \mathrm{~cm}$, estaba fabricado con silicio amorfo y utilizaba como material luminiscente Ioduro de cesio. La eficiencia de detección cuántica de este detector era mayor del 70\%.

La intervención se llevó a cabo mediante tramos de escopia continua, con un kilovoltaje medio de 80 $\mathrm{kV}$ en tasa de dosis normal, y adquisición de imágenes (15 imágenes/s), igualmente en tasa de dosis normal. El kilovoltaje se ajustaba automáticamente mediante el control automático de brillo, optimizado exclusivamente para intervencionismo pediátrico. La filtración del equipo era de $4.1 \mathrm{~mm}$ de $\mathrm{Al}$, con una filtración adicional de $0.2 \mathrm{~mm}$ de $\mathrm{Cu}$.

En los tramos de escopia sólo se utilizó la proyección posteroanterior con una distancia focodetector de $100 \mathrm{~cm}$, foco-piel de $65 \mathrm{~cm}$ y con un tamaño de campo de $20 \mathrm{~cm}$. La adquisición de imágenes se efectuó igualmente con la misma proyección posteroanterior. Únicamente en dos pacientes, una de las series se realizó con una única proyección a $45^{\circ}$. En todos los estudios se utilizó parrilla antidifusora debido al tamaño de los pacientes.

\section{Tasa de dosis vs. Calidad de imagen}

Una vez establecidos los niveles de referencia, se evaluaron, para las dos tasas de dosis implementadas en el equipo, el PDA para diferentes espesores de metacrilato y la calidad de imagen, con el fin de protocolizar el uso habitual de la baja tasa en este tipo de exploración. Para cuantificar la calidad de imagen se utilizó el test de Leeds TO.10, y se determinaron las curvas del umbral de detectabilidad contraste-detalle, que relacionan el índice umbral de detección de un objeto, con un tamaño determinado $\mathrm{H}_{\mathrm{T}}$, con la raíz cuadrada del área de ese objeto. En su cálculo se siguieron las recomendaciones de la bibliografía ${ }^{8}$, pero no se normalizó por un kerma de entrada de referencia, con el fin de evidenciar las diferencias debidas a la tasa de dosis. Cuanto mayor es el índice umbral, menos contraste es necesario para ver un objeto de ese tamaño. En la fase prospectiva, de acuerdo con el clínico intervencionista, y después de evidenciar conjuntamente que no existía una afectación limitante en la calidad de la imagen clínica, se empleó baja tasa de dosis para el desarrollo de los estudios, manteniendo el resto de parámetros fijos. 


\section{Dosimetría por termoluminiscencia}

La dosis en gónadas se midió con una matriz de 4 dosímetros termoluminiscentes de fluoruro de litio TLD-100 (Thermo Electron Corp.), de dimensiones $3.17 \times 3.17 \times 0.89 \mathrm{~mm}$, pertenecientes al mismo lote. Estas matrices se colocaron en la parte inferior de cada testículo, así como en la parte inferior y superior del protector gonadal y sobre la piel, en la zona lumbar izquierda a la altura del riñón. La lectura de la dosis se realizó con un contador manual HARSHAW modelo 3500 (Thermo Electron Corp.). Los dosímetros se calibraron en el mismo equipo donde se llevó a cabo el estudio y con técnicas similares a las utilizadas en condiciones clínicas. La calibración se realizó mediante la intercomparación con una cámara de ionización Radcal modelo 10X5$60 \mathrm{E}$ asociada a un electrómetro Radcal 9015 calibrados por el Centro Nacional de Dosimetría. La incertidumbre estimada de las medidas con los TLD's era inferior al 30\% con un nivel de confianza del 95\%.

\section{Medida del producto dosis área (PDA)}

Para estimar el PDA, el equipo disponía de una cámara de transmisión Diamentor M4KDK (PTW Freiburg, Alemania), calibrada en origen, y que fue verificada midiendo el PDA con el mismo conjunto cámara electrómetro que los TLD`s, para diferentes calidades clínicas del haz. La incertidumbre de la medida era inferior al 10\% con un nivel de confianza del 95\%.

\section{Medida de densidades ópticas relativas}

En este tipo de intervenciones, en las que sólo se utiliza la proyección posteroanterior, la colocación de una placa a la entrada del haz en el paciente nos va a permitir recoger todo el campo de radiación incidente. Se utilizaron películas Kodak X-Omat V de 32.5 x $39.5 \mathrm{~cm}$. La película se proceso automáticamente en una reveladora AGFA Curix HT-330 U sometida a un programa de control de calidad. Posteriormente, la placa se digitalizó utilizando un escáner-densitómetro digital (Vidar System Corp.), y se analizó con el programa de análisis digital Osiris 3.1. Con la adecuada calibración de las peliculas ${ }^{9}$ se estimó el peso relativo de las diferentes proyecciones. Además, se realizaron lecturas densitométricas con un densitómetro X-Rite 331 calibrado para verificar estos valores.

\section{Estimación del riesgo radiológico}

Para obtener la dosis en gónadas, se asumió que la dosis en los testículos es la misma que la medida en los TLD's situados en su parte inferior. En el estudio retrospectivo, se calculó a partir de la correlación lineal entre el PDA y el valor de la dosis medida en los testículos en el estudio prospectivo. Una vez obtenida, se realizó una estimación del riesgo de aparición de efectos hereditarios multiplicando dicha dosis por el factor de riesgo $1 \cdot 10^{-2} \mathrm{~Gy}^{-1}$ recogido en la ICRP-60 y $2.4 \cdot 10^{-2} \mathrm{~Gy}^{-1}$ en la NRPB-R260 para una población en edad reproductiva.

El PDA posibilita la estimación de la energía impartida al paciente, y por tanto, la dosis efectiva y el detrimento estocástico total debido a la radiación ionizante. Como primer paso para la estimación, a partir de las lecturas de los PDA, se utilizaron las tablas del NRPB-SR262 ${ }^{10}$ para obtener la dosis individual asignada a cada órgano. Estas tablas estiman las dosis mediante simulación de Montecarlo para un maniquí antropomórfico adulto y diferentes proyecciones estándar, dependiendo de la técnica utilizada y de la filtración total del equipo. De las proyecciones estándar que ofrece la NRPB-SR262, se eligieron las de "Riñón PA", "Duodeno PA" y "Cadera Izquierda" pesadas en una proporción de 4.25:5:4.25 respectivamente, como las que más se ajustaban a nuestras exploraciones (Fig. 2). Se tomaron los datos correspondientes a dichas proyecciones para un potencial medio aplicado de 80 $\mathrm{kV}$ y una filtración total de $5 \mathrm{~mm}$ de $\mathrm{Al}$. Los cálculos se verificaron utilizando el software EffDose versión $1.04^{11}$. El uso de tablas para adultos se debe a la inexistencia de tales proyecciones en la publicación NRPB-R27912 para pacientes pediátricos, asumiendo dicha publicación un error inferior al 33\% entre el maniquí pediátrico de 15 años y el de adulto. No obstante, tanto el peso medio como la altura de nuestros pacientes (169 cm y $60 \mathrm{Kg}$ ) se encontraban entre los que propone la NRPB-R279 para pacientes pediátricos de 15 años (164 cm y $54 \mathrm{Kg}$ ) y los que propone para adultos la NRPB-SR262 (175 cm y $70 \mathrm{Kg})$.

El cálculo de la dosis efectiva, a partir de las dosis de cada órgano, se realizó siguiendo las recomendaciones de la ICRP-60. Debido a la exclusión del escroto del campo primario y al uso de protector plomado de $1 \mathrm{~mm}$, las dosis medidas en los testícu- 


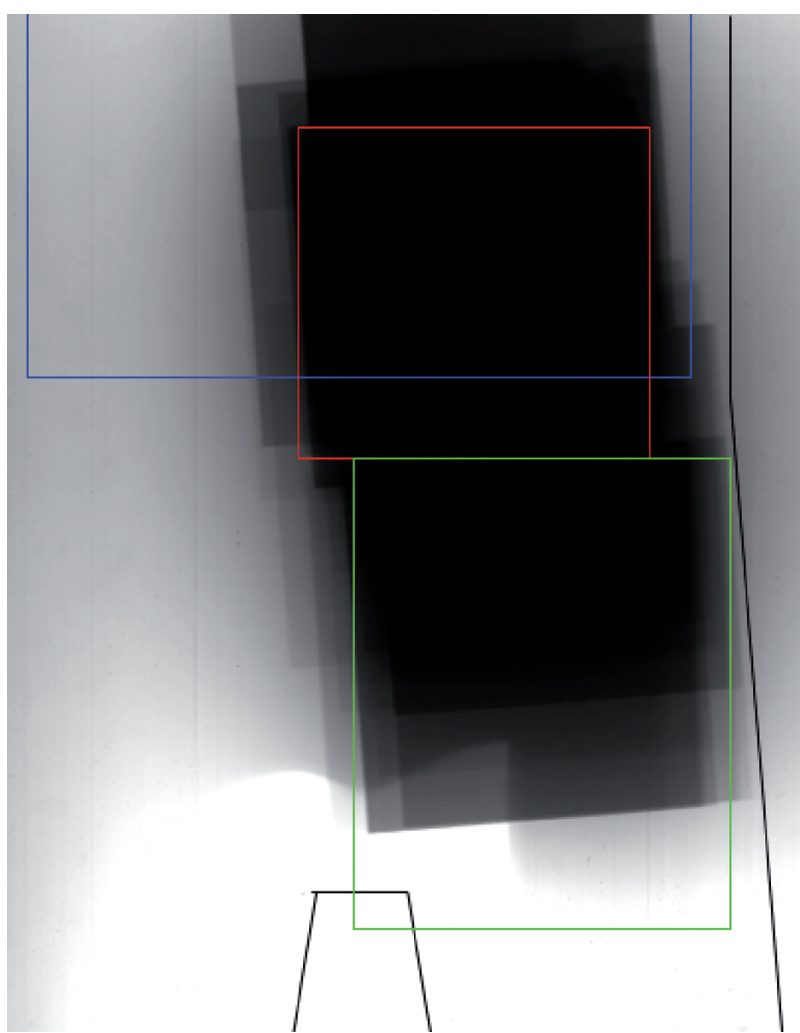

FIGURA 2. Verificación de las proyecciones elegidas.

los son más bajas que los valores obtenidos de las tablas. Por ello, las dosis efectivas para cada paciente se corrigieron reemplazando las dosis calculadas en los testículos con las medidas.

El riesgo total de cáncer fatal se obtiene sumando el riesgo de cáncer fatal para cada órgano. Éste se calcula como el producto de la dosis estimada en cada órgano, por el factor de riesgo de inducción de cáncer fatal por unidad de dosis correspondiente a dicho órgano en hombres con edades entre los 10 y 19 años. Los factores de riesgo, reflejados en la Tabla 1, se determinan a partir de la probabilidad total de inducción de cáncer fatal para una población mixta expuesta a diferentes edades, de la contribución relativa de cada órgano al riesgo de cáncer fatal en una población general, y de la contribución relativa de cada órgano en función del sexo para el modelo de proyección multiplicativo, asumiendo que la contribución relativa de cada órgano no varía con la edad. Además, se utilizaron los factores recogidos en el artículo de Chalmers et al. ${ }^{13}$, calculados con el mismo método, pero a partir de la NRPB-R260 aplicable a la población del Reino Unido.
Tabla 1. Factores de riesgo de inducción de cáncer fatal para hombres entre 10 y 19 años. (x $10^{-6} \mathrm{mSv}^{-1}$ )

\begin{tabular}{lcc}
\hline & ICRP-60 & NRPB-R260 \\
\hline Vejiga & 5.92 & 10.80 \\
Médula ósea & 7.48 & 10.80 \\
Superficie ósea & 0.64 & 0.90 \\
Colon & 7.69 & 5.40 \\
Hígado & 1.92 & 2.70 \\
Pulmón & 11.50 & 20.70 \\
Esófago & 3.17 & 1.80 \\
Piel & 0.26 & 0.27 \\
Estómago & 15.45 & 6.30 \\
Tiroides & 1.02 & 0.36 \\
Resto & & \\
Riñones & 3.20 & 15.75 \\
Otros & 3.20 & 15.75 \\
Total & 6.14 & 9.10 \\
\hline
\end{tabular}

\section{Análisis Estadístico}

Debido a la distribución no normal de las variables analizadas, las diferencias significativas entre ambos estudios se verificaron mediante el Test de MannWhitney. Para los cálculos estadísticos se utilizó el programa SSPS versión 13.0 para Windows. Las incertidumbres en la medida de los TLD se obtuvieron siguiendo las recomendaciones de la bibliografia ${ }^{14}$.

\section{RESULTADOS}

En la Figura 3 se presentan los tiempos de escopia de los 25 casos que formaron parte del estudio completo. No existía una correlación significativa, pero si tendencia a disminuir.

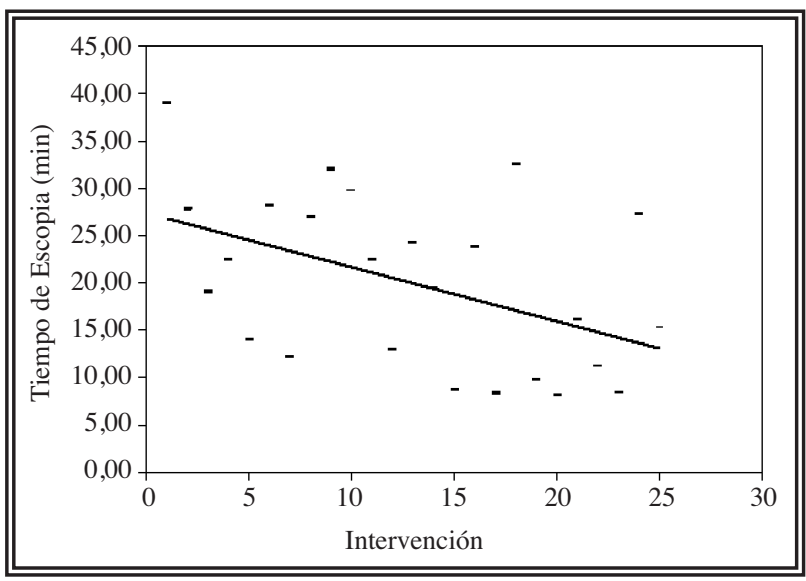

FIGURA 3. Tiempos de escopia ordenados por la fecha de la intervención. $n=25, r=0.47, p<0.01$. 
La Figura 4 muestra la relación entre las tasas de PDA normal y baja, tanto de cine como de escopia, medidas para diferentes espesores de metacrilato. Había una reducción de un $46 \%$ si se usaba cine en baja tasa en lugar de en tasa normal. La reducción llegaba al 56\%, en el caso de modificar la tasa de escopia.

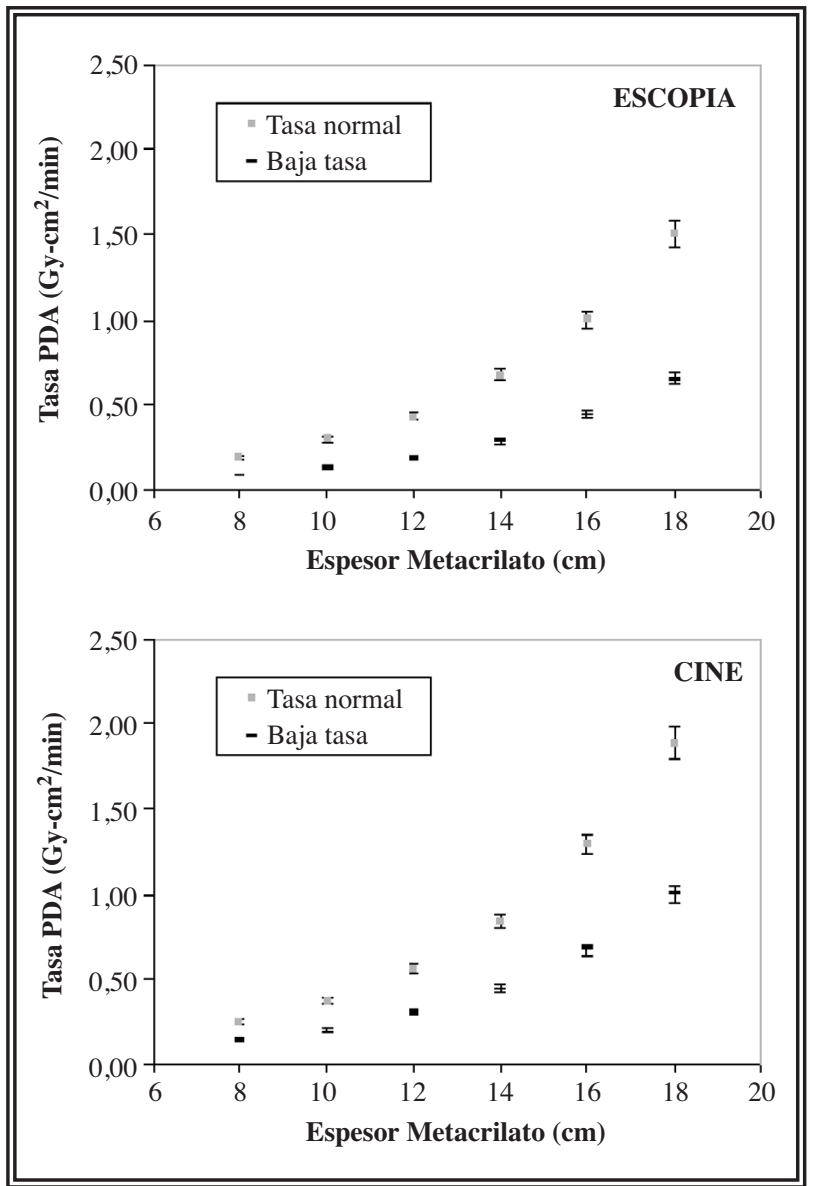

FIGURA 4. Tasas de PDA para diferentes espesores de un maniqui de metacrilato.

En la Figura 5 se muestran las curvas del umbral de detectabilidad contraste-detalle del test de Leeds TO.10 en función de la tasa de dosis utilizada. El aumento del ruido de la imagen por el uso de la baja tasa de dosis supone una pérdida de contraste y calidad en la imagen. Sin embargo, el médico intervencionista no la consideró relevante para la práctica clínica en este procedimiento.

En la Figura 6 aparecen los valores de las tasas de PDA total en función del coeficiente de masa corporal de los pacientes para ambos estudios. No se observó una correlación equivalente a la que se obtuvo con el

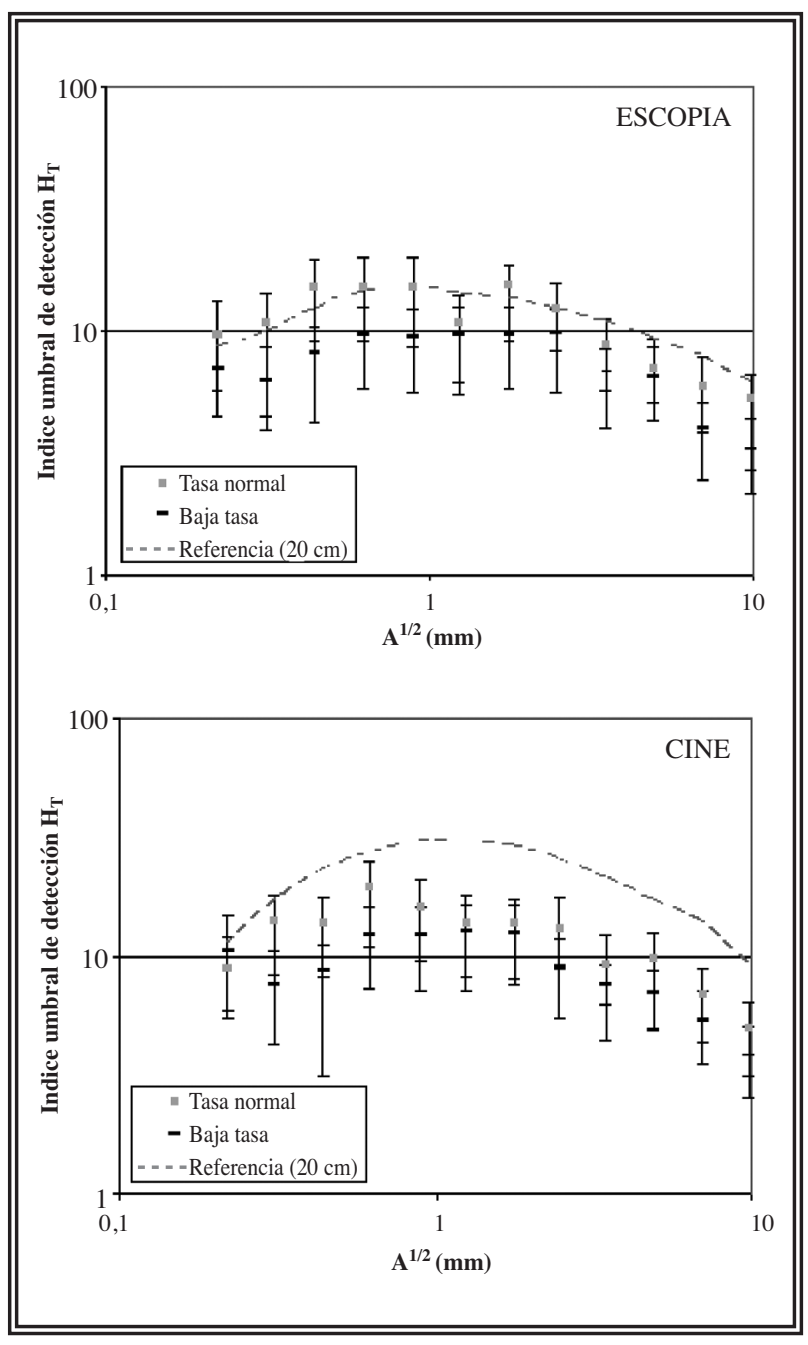

FIGURA 5. Curvas umbral de sensibilidad contraste-detalle.

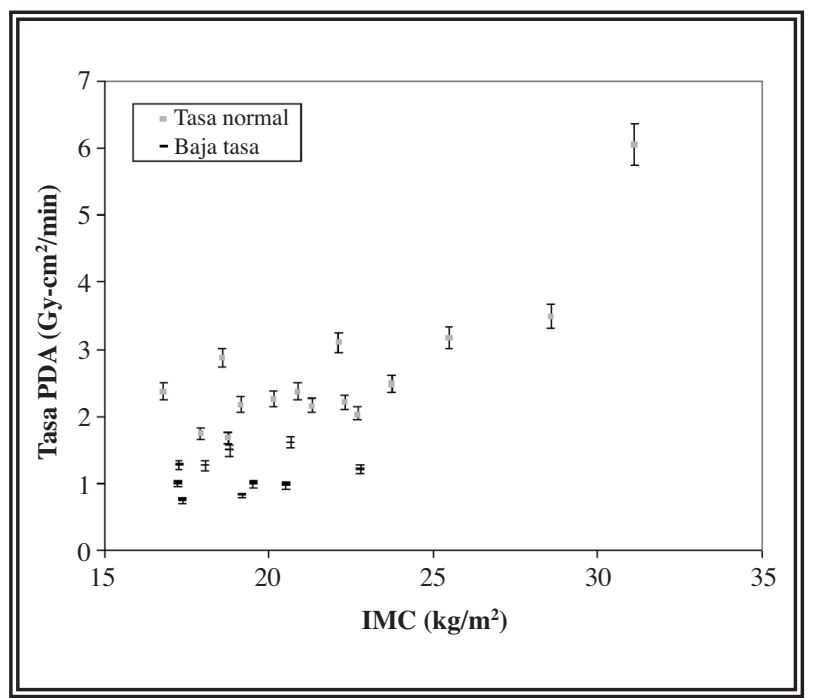

FIGURA 6. Tasa de PDA en función del Índice de Masa Corporal. 
maniquí. Esto era esperable, puesto que el coeficiente de masa corporal tampoco tiene una correlación lineal con el espesor, se superponen tramos de escopia y de cine, y puede haber variaciones en la tasa, debido a la presencia del protector plomado y a que el haz atraviesa tejidos de densidades variables a lo largo del desarrollo de la intervención. Si se observó una diferencia significativa entre la tasa para las dos series en estudio $(\mathrm{p}<0.001)$, reduciéndose un $57 \%$ en caso de utilizar el modo de baja tasa.

Los valores de las variables objeto de análisis: número de pacientes, edad, peso, tiempo de escopia, número de imágenes, PDA, dosis en gónadas, riesgo de aparición de efectos hereditarios, dosis efectiva y riesgo de cáncer fatal se representan en la Tabla 2. La edad media de los pacientes, el tiempo de escopia y el número de imágenes son menores para el estudio prospectivo, aunque sin significación estadística. La disminución en el resto de variables, si tiene significación estadística. Sin embargo, mientras la reducción en el peso de los pacientes no llega al 13\% (p<0.05), la del PDA y por tanto la de la dosis efectiva y la del riesgo estocástico es superior al 73\% $(\mathrm{p}<0.001)$

En la Tabla 3 se muestra una comparación entre los valores que obtuvimos en nuestro estudio y los publicados hasta la fecha para pacientes adultos.

Tabla 2. Resumen de resultados

\begin{tabular}{|c|c|c|c|c|c|c|c|c|}
\hline & \multicolumn{4}{|c|}{ Estudio Retrospectivo } & \multicolumn{4}{|c|}{ Estudio Prospectivo } \\
\hline & Media & Mediana & $1^{\mathrm{er}}-3^{\mathrm{er}}$ cuartil & Mín-Máx & Media & Mediana & $1^{\mathrm{er}}-3^{\mathrm{er}}$ cuartil & Mín-Máx \\
\hline Edad & 14.3 & 15 & $14-15$ & $10-18$ & 13.9 & 15 & $12-15$ & $10-16$ \\
\hline Peso & 63.5 & 66 & $56-70.7$ & $44.5-90$ & 55.7 & 58.2 & $45.1-64.7$ & $39.3-69$ \\
\hline Tiempo de Escopia (min) & 22.6 & 22.5 & $16.5-27.9$ & $8.7-39.1$ & 17 & 15.7 & $12.2-21.8$ & $8.2-32.5$ \\
\hline $\mathrm{N}^{\circ}$ Total de Imágenes & 798 & 806 & $466-991$ & $173-1772$ & 603 & 578 & $527-666$ & $519-773$ \\
\hline $\mathrm{PDA}\left(\mathrm{Gy} \cdot \mathrm{cm}^{2}\right)$ & 59.67 & 58.76 & $38.41-74.78$ & $19.70-117.52$ & 16.20 & 13.10 & $12.18-21.38$ & $8.41-52.33$ \\
\hline Dosis Gónadas (mGy) & 0.30 & 0.29 & $0.19-0.37$ & $0.10-0.59$ & 0.12 & 0.10 & $0.07-0.16$ & $0.05-0.26$ \\
\hline Riesgo de Efectos Hereditarios NRPB-R260 (x10-6) & 7.1 & 7.0 & $4.6-9.0$ & $2.4-14.1$ & 2.9 & 2.4 & $1.7-3.9$ & $1.2-6.3$ \\
\hline Riesgo de Efectos Hereditarios ICRP-60 (x10-6) & 3.0 & 2.9 & $1.9-3.7$ & $1.0-5.9$ & 1.2 & 1.0 & $0.7-1.6$ & $0.5-2.6$ \\
\hline Dosis Efectiva (mSv) & 8.8 & 8.7 & $5.6-11.0$ & $2.9-17.3$ & 2.4 & 1.9 & $1.8-3.1$ & $1.3-7.7$ \\
\hline Riesgo de Cáncer Fatal NRPB-R260 (x10-6) & 1636 & 1611 & $1052-2050$ & $540-3221$ & 444 & 359 & $334-586$ & 231-1434 \\
\hline Riesgo de Cáncer Fatal ICRP-60 (x10-6) & 792 & 780 & $510-993$ & 261-1559 & 215 & 174 & $162-284$ & $112-694$ \\
\hline
\end{tabular}

Tabla 3. Comparativa con estudios anteriormente publicados para pacientes adultos. Los valores vienen clasificados en media, mediana, (1er - 3er cuartil) y (mínimo - máximo)

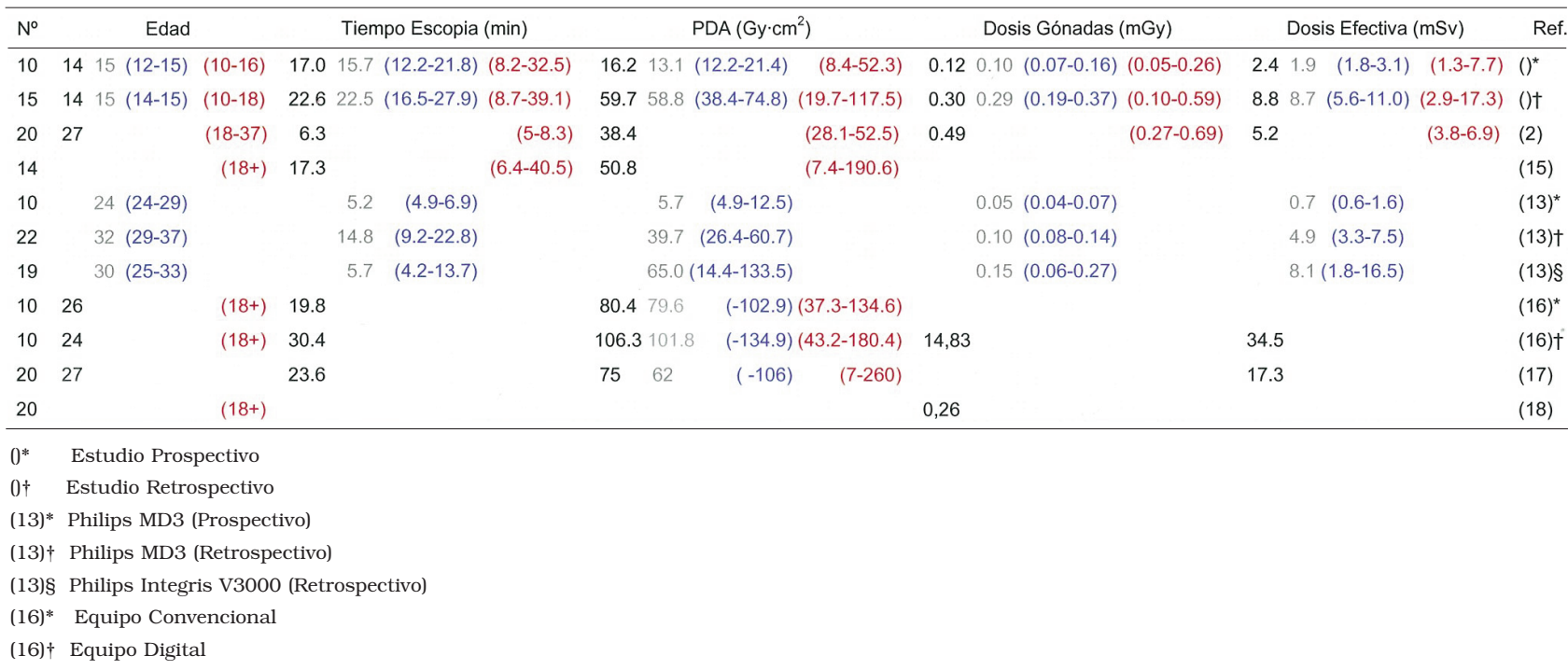




\section{DISCUSIÓN}

En muchos centros hospitalarios se elige la embolización del varicocele como terapia, en lugar de técnicas quirúrgicas, por tratarse de un procedimiento mínimamente invasivo y que proporciona mayor comodidad al paciente. Sin embargo, pocos clínicos contemplan los riesgos radiológicos asociados a este tipo de intervención, y solo algunos trabajos han estimado los riesgos hereditarios asociados a las dosis recibidas en gónadas y el riesgo de inducción de cáncer para pacientes adultos, sin existir referencias para pacientes pediátricos.

Estudios sobre pacientes adultos como los de Chalmers et al. y Gazzera et al. muestran tiempos de escopia medios inferiores a los obtenidos en el nuestro, si bien, del análisis individual de los tiempos recogidos, observamos una tendencia a la baja durante los tres años, debido a la experiencia adquirida en este tipo de procedimiento por el intervencionista pediátrico que llevó a cabo todos los tratamientos. Esta diferencia de tiempos se manifestaría en un mayor valor del PDA en nuestro estudio, pero el uso de técnicas de reducción de dosis específicas del equipo utilizado minimizan este incremento. El equipo de rayos $\mathrm{X}$ dispone de control automático de brillo, optimizado para pacientes pediátricos, lo cual contribuye, considerando la ventaja del tamaño de estos pacientes, a una reducción del PDA. Sin embargo, aunque se utilizaba el control automático de brillo, no se utilizaba la baja tasa de dosis como herramienta que permite optimizar aún más la dosis en función del ruido de la imagen. La introducción del uso de baja tasa supuso una disminución media de la tasa de PDA de un 57\%, y por tanto una reducción de la dosis y del riesgo radiológico por unidad de tiempo del mismo orden, sin un deterioro limitante de la calidad de imagen. En consecuencia, el futuro debe encaminarse hacia la adopción de medidas de reducción de dosis, tanto por optimización del tiempo total de escopia (método de embolización adecuado, experiencia, etc.), como por el uso habitual del modo de escopia de tasa reducida. Otros métodos de reducción como disminuir la distancia foco-piel, utilizar campos más colimados, etc., también deben ser valorados.

En el estudio, alguno de los pacientes llega a recibir una dosis efectiva de $17.3 \mathrm{mSv}$, y en el peor caso, el riesgo estimado de inducción de cáncer fatal es de 3221 casos por millón. Sin embargo, los valores reflejados por las medianas son inferiores a la mitad del valor máximo debido a una mayor frecuencia de las dosis pequeñas. Esto supone un incremento promediado del $0.16 \%$ sobre el $20 \%$ de incidencia natural de cáncer ${ }^{19,20}$ para el estudio retrospectivo, y un $0.04 \%$ para el prospectivo. No se debe olvidar que, en caso de recidiva, puede tener lugar una nueva intervención con el consiguiente incremento de dosis. Además, este tipo de intervención es clínicamente más compleja que la inicial debido a la necesidad de realizar un estudio vascular exhaustivo de la zona para determinar las venas afectadas, con lo que se requieren tiempos de escopia y dosis mayores. En nuestro caso, 2 de los primeros pacientes del grupo retrospectivo requirieron de una segunda intervención. En ambas, el tiempo de escopia aumentó en un factor entre 2 y 3 , y el PDA en un factor próximo a 4 respecto de la primera embolización. La dosis total como consecuencia de la doble intervención ascendió a $11.2 \mathrm{mSv}$ y $14 \mathrm{mSv}$ respectivamente.

A la hora de comparar estas dosis efectivas con las de otras publicaciones, se deben valorar las diferentes elecciones en las proyecciones utilizadas. Chalmers et al. utilizan las mismas proyecciones que nuestro trabajo, pero con pesos diferentes, obteniendo estimaciones ligeramente inferiores. Ruiz Cruces et al. utilizan la proyección "Pelvis PA" para su estimación. Esta elección comporta un aumento significativo de la dosis efectiva estimada (superior al 100\%), al elegir una región más amplia que no ajusta los órganos de riesgo irradiados. En cuanto a la estimación del riesgo de cáncer fatal, la diferencia con Chalmers et al., además de por la elección de pesos en las proyecciones, se debe a la evaluación de diferentes grupos de edad con sus correspondientes factores. Para la estimación de Ruiz-Cruces et al., ni el método utilizado para estimar la dosis efectiva (proyección utilizada) ni el factor utilizado para su cálculo (independiente de órgano, edad, y sexo) permiten comparar resultados.

Las estimaciones de la dosis efectiva y del riesgo fatal de cáncer tienen una precisión relativa, que depende del ajuste entre los tamaños de campo y las posiciones del tubo de rayos $\mathrm{X}$ a lo largo de la intervención, y las proyecciones estándar publicadas en la NRPB-SR262. Además hay que considerar que la anatomía de los pacientes pediátricos es más variable que la de los adultos en cuanto a peso y altura, por lo que su adaptación a un tamaño único de maniquí para la simulación es menos precisa. A 
esta incertidumbre hay que sumar la asociada a los factores de riesgo de cáncer necesarios para su estimación. Los factores utilizados han sido calculados mediante protocolos internacionales de estimación de riesgo estocástico, basados en estudios estadísticos de aumento de incidencia de cáncer en poblaciones irradiadas a altas dosis y no a partir de resultados de seguimiento de pacientes de varicocele irradiados clínicamente. Chalmers et al. especifican que la estimación del riesgo de cáncer a partir de sus factores lleva asociada una incertidumbre de hasta el 50\%. La ICRP-60 también habla de incertidumbres considerables en los factores de riesgo para los diferentes órganos como consecuencia de la dosimetría, la tasa de dosis, la latencia, la forma de la relación dosis-respuesta y, naturalmente, de las incertidumbres en los propios datos de base. Sin embargo, son las mejores estimaciones de riesgo disponibles. Las diferencias entre los riesgos de cáncer fatal, según se utilicen unos factores de riesgo $u$ otros, se deben a que se han estimado para diferentes poblaciones. La NRPB-R260 utiliza los datos referidos a la población del Reino Unido, mientras que la ICRP-60 les da un valor promedio en el que se contemplan las diferentes poblaciones mundiales.

El detrimento total debido la radiación debe incluir, además del riesgo de cáncer mortal, las probabilidades pesadas de cánceres no mortales y los riesgos hereditarios severos. Si se asume que el factor para el riesgo de cáncer no mortal y de efectos hereditarios graves es, al igual que el de cáncer fatal, aproximadamente 1.5 veces mayor para nuestro grupo de edad que para el promedio, según el modelo multiplicativo, este detrimento supone una adición de 35 casos por millón y mSv sobre el riesgo de cáncer fatal para el riesgo estocástico global.

Las dosis en las gónadas y los consecuentes efectos hereditarios son muy bajos comparados con el $6 \%$ de incidencia natural de defectos congénitos ${ }^{21}$, incluso asumiendo una incertidumbre en las medidas con TLD's de hasta el 30\%. La inclusión del protector gonadal disminuye significativamente la dosis testicular si el haz incide directamente sobre las gónadas. Este efecto no es relevante en el caso de que el haz no incida sobre el protector, como hemos observado de las lecturas dosimétricas con TLD situados a ambos lados del mismo durante las intervenciones.
De las dosis estimadas en piel y gónadas se pueden descartar efectos deterministas para ambos órganos, en los procedimientos habituales y en ausencia de complicaciones, de acuerdo a la catalogación de la embolización percutánea de varicocele por parte de la ICRP-85 como técnica de dosis media. Estos efectos presentan un umbral de dosis de 2 Gy (eritemas en piel) y 150 mGy (esterilidad temporal en testículos), valores muy por encima de los máximos medidos; 250 mGy de media en la piel, en la zona lumbar izquierda y 0.6 mGy en los testículos.

\section{CONCLUSIONES}

Las embolizaciones de varicocele se llevan a cabo en pacientes jóvenes con una esperanza de vida normal, a diferencia de la mayoría de intervenciones vasculares. La posibilidad de recidiva en la embolización es similar en porcentaje a la que se asocia al resto de técnicas intervencionistas, y el riesgo de complicaciones severas es poco común para todas las intervenciones quirúrgicas ${ }^{22}$, por lo que el riesgo estocástico radiológico, a pesar de las incertidumbres asociadas a los diferentes métodos de cálculo, se hace relevante. Es por tanto de vital importancia realizar esfuerzos para optimizar la exposición.

La dosis de radiación está optimizada cuando la imagen se conforma con el mínimo de radiación necesaria para tener una calidad diagnóstica adecuada. Esto requiere equipo y entrenamiento específicos, y controles constantes. Con la tecnología actual, la dosis puede minimizarse poniendo especial atención en los medios de optimización que incorpora el equipo y en el procedimiento utilizado, así como en una adecuada formación y experiencia tanto del técnico responsable del funcionamiento del equipo radiológico como del médico intervencionista. Todos los sistemas de reducción de dosis disponibles en el equipo radiológico deben ser conocidos por los operadores y ser aplicados durante la práctica habitual de la intervención.

No se puede descartar la incidencia directa del haz sobre los testículos durante el procedimiento, por lo que se recomienda, si no se utiliza control automático de brillo, el uso de protector gonadal para reducir la dosis en estos órganos durante la exposición. Sin embargo, la inclusión del protector dentro del campo de radiación en equipos con control automático de brillo supone un aumento considerable de la tasa de PDA, y por tanto, de la dosis en las zonas no protegidas, por lo que se debe valorar su uso particular. 
Es necesario que los médicos prescriptores e intervencionistas conozcan las dosis asociadas a las embolizaciones de varicocele y sus efectos potenciales, y justifiquen la intervención correctamente, valorando las posibles alternativas. También legalmente se encuentran obligados a facilitar esta información al paciente, de forma que dentro del consentimiento informado se recoja su conformidad en afrontar los riesgos radiológicos derivados.

\section{Agradecimientos}

Queremos agradecer la colaboración de la Sección de Hemodinámica del Servicio de Cardiología y de Urología del Servicio de Cirugía Pediátrica del Hospital Materno-Infantil del Gregorio Marañón.

\section{REFERENCIAS}

1. Skoog SJ, Roberts KP, Goldstein M, Pryor JL. The adolescent varicocele: What's new with an old problem in young patients? Pediatrics. 1997;100(1);112-127.

2. Gazzera C, Rampado O, Savio L, Di Bisceglie C, Manieri C, Gandini G. Radiological treatment of male varicocele: technical, clinical, seminal and dosimetric aspects. Radiol Med. 2006;111 (3):449-458.

3. Lima SS, Castro MP, Costa OF. A new method for the treatment of varicocele. Andrologica. 1978;10(2):103-106.

4. Recomendaciones de la Comisión Internacional de Protección Radiologica. ICRP Publication 60. Ann ICRP 1990.

5. Estimates of late radiation risks to the UK population. Documents of the NRPB. Chilton, UK: National Radiological Protection Board, 1993.

6. Robb JD. Estimates of radiation detriment in a UK population. NRPB-R260. Chilton, UK: National Radiological Protection Board, 1994.

7. Avoidance of radiation injuries from medical interventional procedures. ICRP Publication 85. Ann ICRP 2000.

8. Evans DS, Mackencie A, Lawinski CP, Smith D. Threshold contrast detail detectability curves for fluoroscopy and digital adquisition using modern image intensifier systems. Brit $\mathrm{J}$ Radiol. 2004 77(921):751-758.

9. Vañó E, Guibelalde E, Fernández JM, Gonzalez L, Ten JI. Patient dosimetry in interventional radiology using slow films. Brit J Radiol. 1997;70:195-200.

10. Hart D, Jones DG, Wall BF. Normalised organ doses for medical x-ray examinations calculated using monte carlo techniques. NRPB-SR262. Chilton, UK: National Radiological Protection Board, 1994
11. Effdose software (Version 1.04). X-Ray radiography dosimetry program using NRPB-SR262. National Board of Health, Denmark. National Institute of Radiation Hygiene, 1995.

12. Coefficients for estimating effective doses from paediatric $\mathrm{x}$-ray examinations. NRPB-R279. Chilton, UK: National Radiological Protection Board. March 1996.

13. Chalmers N, Hufton AP, Jackson RW, Conway B. Radiation risk estimation in varicocele embolization. Brit J Radiol. 2000; 73 293-297.

14. National protocol for patient dose measurements in diagnostic radiology. Chilton, UK: National Radiological Protection Board 1992.

15. Miller DL, Balter S, Cole PE, Lu HT, Schueler BA, Geisinger M, et al. Radiation doses in interventional radiology procedures: The RAD-IR study. J Vasc Interv Radiol. 2003;14(6):711-727.

16. Ruiz-Cruces R, Pérez-Martínez M, Martín-Palanca A, Flores A Cristófol J, Martínez-Morillo M, et al. Patient dose in radiologically guided interventional vascular procedures: Convencional versus digital systems. Radiology. 1997;205(2):385-393.

17. Ruiz Cruces R, García-Granados J, Diaz Romero FJ, Hernández Armas J. Estimation of effective dose in some digital angiographic and interventional procedures. Brit J Radiol. 1998 71(841):42-47.

18. Walsh PC, White RI Jr. Ballon occlusion of the internal spermatic vein for the treatment of varicoceles. JAMA. 1981;246 (15):1701-1702.

19. National Research Council. Health effects exposure to low leve of ionizing radiation. BEIR V. Washington, DC: National Academy Press; 1990.

20. U.S. Cancer Statistics Working Group. United States Cancer Statistics: 1999-2002 Incidence and mortality web-based report. Atlanta: U.S. Department of Health and Human Services, Centers for Disease Control and Prevention and National Cancer Institute; 2005.

21. Hall EJ. Hereditary effects of radiation. In: Hall EJ, ed. Radiobiology for the Radiologist. Philadelphia, PA: IB Lippinett CO; 1994.

22. Beutner S, May M, Hoschke B, Helke C, Lein M, Roigas J, et al. Treatment of varicocele with reference to age: a retrospective comparison of three minimally invasive procedures. Surg Endosc. 2007;21(1):61-65.

Correspondencia autor: Dr. J.A. Calama Santiago Servicio de Dosimetría y Radioprotección

Hospital General Universitario Gregorio Marañón Doctor Esquerdo, 46 - 28007 Madrid

Tel: 915868182

E-mail autor: juan_calama@hotmail.com

Información artículo: Original - Urología Pediátrica

Trabajo recibido: febrero 2008

Trabajo aceptado: abril 2008 